\title{
Lagartos de áreas de Cerrado na Reserva Biológica Unilavras-Boqueirão, Ingaí, sul de Minas Gerais, Brasil
}

\author{
Iara Alves Novelli, ${ }^{1,3}$, Priscila da Silva Lucas ${ }^{2}$, Ramon Gomes de Carvalho', \\ Rodrigo Costa Santos ${ }^{1}$ \& Bernadete Maria de Sousa ${ }^{2}$
}

${ }^{1}$ Laboratório de Zoologia, Centro Universitário de Lavras - UNILAVRAS, Rua Padre José Poggel, 506, Bairro Centenário, CEP 37200-000, Lavras, MG, Brasil

${ }^{2}$ Laboratório de Herpetologia, Departamento de Zoologia, Instituto de Ciências Biológicas, Universidade Federal de Juiz de Fora - UFJF, Campus Universitário, s/n, Martelos, CEP 36036-900, Juiz de Fora, MG, Brasil

${ }^{3}$ Autor para correspondência: Iara Alves Novelli, e-mail: iaranovelli27@gmail.com

NOVELLI, I.A., LUCAS, P.S., CARVALHO, R.G., SANTOS, R.C. \& SOUSA, B.M. Lizards of the Cerrado areas in the Reserva Biológica Unilavras-Boqueirão, Ingaí, Minas Gerais Southern, Brazil. Biota Neotrop. 12(3): http://www.biotaneotropica.org.br/v12n3/en/abstract?inventory+bn00312032012

\begin{abstract}
Studies with emphasis on assessment and quantification of species biological diversity should be recognized as important tools in the process of knowledge, especially where basic data are scarce. This study was conducted at the Reserva Biológica Unilavras-Boqueirão (21 ${ }^{\circ} 20^{\prime} 47^{\prime \prime} \mathrm{S}$ and $44^{\circ} 59^{\prime} 27^{\prime \prime} \mathrm{W}$ ) inserted in the Cerrado, Ingaí municipality, southern Minas Gerais, southeastern Brazil. We aimed to characterize and understand local lizard species. Sampling occurred between June 2008 and February 2010 in weekly excursions during the day in the field. Captures were carried out by means of pitfall traps, distributed in six sets, three in Cerrado sensu stricto and three in the gallery forest, making a sampling effort of 6120 buckets per day. Animals viewed, occasionally found or collected by third parties were also included in the sample. A total of 10 lizard species from seven families were captured. The richest vegetation type was the Cerrado sensu stricto with nine species. Only Heterodactylus imbricatus was restricted to gallery forests, where pitfalls sampled $74.12 \%$ of lizards captured. The lizard fauna is well represented in the area with species typical of open areas as Ameiva ameiva, Polychrus acutirostris and Mabuya frenata. The species Urostrophus vautieri and Heterodactylus imbricatus, not yet reported for the Cerrado, were also found in the area.
\end{abstract}

Keywords: squamata, Cerrado, gallery forest, rocky fields, Minas Gerais state.

NOVELLI, I.A., LUCAS, P.S., CARVALHO, R. G., SANTOS, R.C. \& SOUSA, B. M. Lagartos de áreas de Cerrado na Reserva Biológica Unilavras-Boqueirão, Ingaí, Sul de Minas Gerais, Brasil. Biota Neotrop. 12(3): http://www.biotaneotropica.org.br/v12n3/pt/abstract?inventory+bn00312032012

Resumo: Estudos com ênfase em levantamentos e quantificação da diversidade biológica das espécies devem ser reconhecidos como ferramentas importantes no processo de produção de conhecimento básico principalmente onde dados sobre a fauna são escassos. O presente estudo objetivou caracterizar a fauna de lagartos da Reserva Biológica Unilavras-Boqueirão ( $21^{\circ} 20^{\prime} 47^{\prime}$ S e $\left.44^{\circ} 59^{\prime} 27^{\prime} \mathrm{W}\right)$, inserida no domínio do Cerrado, situada no município de Ingaí, Sul de Minas Gerais, Sudeste do Brasil. A amostragem ocorreu entre junho de 2008 a fevereiro de 2010, em excursões semanais no período diurno em campo. As capturas foram realizadas por meio de armadilhas de queda, distribuídas em seis conjuntos, três em área de Cerrado sensu stricto e três em Mata de galeria, perfazendo um esforço amostral de 6.120 baldes/dia. Animais visualizados, encontrados ocasionalmente ou coletados por terceiros também foram incluídos na amostragem. Um total de 10 espécies de lagartos foram capturados, distribuídos em sete famílias. A fitofisionomia onde foi amostrado o maior número de espécies foi o Cerrado sensu stricto, com nove espécies. Apenas Heterodactylus imbricatus esteve restrita à fitofisionomia de mata de galeria, onde as armadilhas de queda capturaram $74,12 \%$ dos lagartos. A fauna de lagartos é representada por espécies típicas de áreas abertas como Ameiva ameiva, Polychrus acutirostris e Mabuya frenata e espécies florestais como Urostrophus vautieri e Heterodactylus imbricatus até então não registradas para o Cerrado. Palavras-chave: squamata, Cerrado, mata de galeria, campos rupestres, Minas Gerais. 


\section{Introdução}

Estudos com ênfase em levantamentos e quantificação da diversidade biológica devem ser reconhecidos como ferramentas importantes no processo de produção de conhecimento básico para subsidiar posteriores pesquisas nas áreas de sistemática, ecologia, biogeografia e biologia da conservação (Heyer et al. 1994). Os lagartos, incluídos na ordem Squamata, apresentam grande diversidade e ampla distribuição geográfica, sendo encontrados em vários habitats e compreendendo cerca de 5079 espécies descritas atualmente (Uetz \& Hallerman 2010). Destas, 241 espécies são de ocorrência natural no Brasil (Bérnils 2010), várias endêmicas e com padrão de distribuição restrito e outras com uma distribuição ampla contínua ou disjunta no território brasileiro.

$\mathrm{O}$ estado de Minas Gerais possui uma ampla área territorial e a uma variedade de ecorregiões (i.e. Mata Atlântica, Caatinga e Cerrado) e de ambientes que os compõem, com possibilidade de abrigar uma rica biodiversidade. As áreas de Cerrado no Estado, que já ocuparam aproximadamente 57\% da extensão territorial, atualmente se encontram em rápido processo de transformação, cedendo espaço para culturas/monoculturas agrícolas e florestais ou para a implantação de atividades agropecuárias (Drummond et al. 2009). Apesar do Cerrado ser reconhecido como um dos 34 hotspots para estudo e conservação da diversidade mundial (Mittermeier et al. 2004), as áreas mineiras deste bioma carecem ainda de uma amostragem sistemática e com cobertura geográfica ampla. Entretanto, alguns estudos sobre conservação, diversidade de répteis (Brites \& Bauab 1988, Assis 1999, Mesquita et al. 2007, Recoder \& Nogueira 2007, São Pedro \& Pires 2009) e distribuição geográfica (Barros Filho et al. 2005, Sousa \& Novelli 2009) existem para estas áreas. Recentemente foram registradas pela primeira no Cerrado as espécies de lagartos Urostrophus vautieri (Santos et al. 2009) e Heterodactylus imbricatus (Novelli et al. 2010), provenientes da Reserva Biológica Unilavras - Boqueirão, que está inserida em uma área prioritária para conservação no Estado (Drummond et al. 2005).

O Cerrado é a segunda ecorregião mais rica em lagartos no Brasil (Rodrigues 2005a, Nogueira et al. 2011), perdendo apenas para a Amazônia. Em Minas Gerais, os dados sobre a fauna de répteis estão restritos a poucas localidades, apesar de 57 espécies de lagartos serem conhecidas para o Estado (Bérnils et al. 2009). Informações biológicas sobre o grupo são escassas, dificultando a definição de áreas prioritárias de preservação de maior interesse específico para os répteis (Drummond et al. 2005). Dessa forma, o presente estudo teve como objetivo registrar as espécies de lagartos que ocorrem em três fisionomias características de uma área de Cerrado no município de Ingaí, sul de Minas Gerais, até então não inventariada em relação a sua fauna de lagartos.

\section{Material e Métodos}

\section{1. Área de estudo}

A Reserva Biológica Unilavras - Boqueirão - RBUB (2120'47' S e $44^{\circ} 59^{\prime} 27^{\prime}$ 'W) está localizada no município de Ingaí, na região sul do Estado de Minas Gerais e ocupa uma área total de 159,5 hectares. As elevações da região variam entre 1100 e 1250 m. A precipitação média anual é de $1.411 \mathrm{~mm}$, com chuvas mal distribuídas durante o ano, predominando no verão, com $66,77 \%$ incidindo entre novembro e fevereiro. $\mathrm{O}$ inverno tem cerca de quatro meses com pequeno déficit hídrico, entre $10 \mathrm{~mm}$ e $30 \mathrm{~mm}$. A temperatura média anual situa-se em torno de $19,3{ }^{\circ} \mathrm{C}$. A área da Reserva enquadra-se como uma das áreas prioritárias para conservação da biodiversidade de Minas Gerais, onde podem ser observadas diversas fitofisionomias que caracterizam o Cerrado, inclusive algumas de baixa representatividade e com pouco conhecimento no território nacional, como os campos rupestres (Pereira \& Volpato 2005). A área da reserva possui cinco nascentes e a paisagem é composta pelas fisionomias vegetacionais de mata de galeria, Cerrado sensu stricto e campo rupestre.

\section{Coleta de dados}

A amostragem da fauna de lagartos foi realizada entre junho de 2008 e fevereiro de 2010, em excursões semanais diurnas. As coletas/capturas foram realizadas em três áreas distintas da RBUB: Cerrado sensu stricto (sítio 01), Mata de Galeria (sítio 02) e Campo Rupestre (sítio 3). Para a amostragem dos espécimes na áreas de Cerrado sensu stricto e em mata de galeria foram instalados no total seis conjuntos de armadilhas de interceptação e queda (Cechin \& Martins 2000, Enge 2001, Bernarde 2008), três conjuntos em cada sítio de coleta. Cada conjunto de armadilhas foi composto por quatro linhas paralelas de $12 \mathrm{~m}$, cada uma com três baldes de $30 \mathrm{~L}$ enterrados, distantes $6 \mathrm{~m}$ um do outro, com cercas direcionadoras em cada uma das linhas, constituída por tela de mosqueteiro. Assim, foram instaladas 36 armadilhas em cada sítio de coleta. $\mathrm{Na}$ área de campo rupestre não foi possível a instalação de armadilhas de interceptação e queda, pois essa fitofisionomia possui um substrato rochoso que não possibilitou a instalação dos baldes de coleta. Em todos os três sítios de amostragem também foram utilizadas armadilhas de cola instaladas em locais estratégicos de provável encontro dos lagartos conforme metodologia proposta por Bauer \& Sadlier (1992) e Zani \& Vitt (1995), durante os períodos de vistoria das armadilhas e dos outros métodos de amostragem. Animais avistados, encontrados ocasionalmente ou coletados por terceiros também foram contabilizados.

Os espécimes capturados foram fotografados e marcados com elásticos coloridos de silicone (Ribeiro \& Sousa 2006) e soltos no seu habitat natural, nos locais onde foram capturados. Espécimes representativos (voucher) foram coletados, mortos com Tiopental, fixados em formaldeído a $10 \%$, conservados em etanol $70 \%$ e depositados na Coleção de Répteis do Laboratório de Zoologia do Centro Universitário de Lavras (CRLZ - UNILAVRAS).

Para quantificar o esforço amostral foram construídas curvas de rarefação de espécies a partir da média de 1.000 aleatorizações dos dias de coleta com o programa EstimateS V.8.2.0 através do método Bootstrap (Colwell 2006) para a totalidade da amostra.

\section{Resultados}

Foram realizadas 85 expedições ao campo e um esforço amostral de 3.060 horas-balde/dia nos sítios 01 e 02 e 765 horas por unidade amostral, totalizando 6.120 horas-balde/dia. Foram capturados no total 85 espécimes de lagartos, pertencentes a 10 espécies (Tabela 1). Destes, 63 espécimes foram capturados em armadilhas de interceptação e queda, 12 em encontros ocasionais, sete por terceiros e três capturados em armadilhas de cola. Os espécimes registrados no presente estudo estão distribuídos em sete famílias: Anguidae (uma espécie), Gymnophthalmidae (duas espécies), Leiosauridae (duas espécies), Polychrotidae (uma espécie), Scincidae (duas espécies), Teiidae (uma espécie) e Tropiduridae (uma espécie) (Tabela 1).

A fitofisionomia que apresentou maior número de espécies foi o Cerrado sensu stricto (sítio 01) com nove espécies registradas: Enyalius bilineatus Duméril \& Bibron, 1837 (Leiosauridae) (Figura 1d), Ameiva ameiva (Linnaeus, 1758) (Teiidae) (Figura 2c), Urostrophus vautieri Duméril \& Bibron, 1837 (Leiosauridae) (Figura 1e), Tropidurus itambere Rodrigues, 1987 (Tropiduridae) (Figura 2d), Polychrus acutirostris Spix, 1825 (Polychrotidae) (Figura 1f), Ophiodes striatus (Spix, 1825) (Anguidae) (Figura 1a), 
Cercosaura ocellata Wagler, 1830 (Gymnophthalmidae) (Figura 1c), Mabuya frenata (Cope, 1862) (Scincidae) e M. dorsivittata Cope, 1862 (Scincidae) (Figura 2a, b). Na Mata de Galeria (sítio 02) foram registradas cinco espécies: E. bilineatus, U. vautieri, M. frenata, M. dorsivittata e Heterodactylus imbricatus Spix, 1825 (Gymnophthalmidae) (Figura 1b); e no campo rupestre (sítio 03) foram registradas três espécies: $M$. frenata, $T$. itambere e $C$. ocellata (Figura 3, Tabela 1).

Um total de 11 indivíduos foram capturados, marcados, medidos, fotografados, identificados e soltos nos locais onde foram encontrados: quatro $P$. acutirostris, um $M$. frenata, dois $U$. vautieri e quatro E. bilineatus. Não foi registrada nenhuma recaptura durante todo período de estudo.

O método que amostrou o maior número de espécies $(n=9)$ e espécimes $(74,12 \%)$ de lagartos foi a armadilha de interceptação e queda. Avistamentos, encontros ocasionais, armadilhas de cola e coleta por terceiros amostraram juntos $25,88 \%$ dos espécimes de lagartos.

A curva de rarefação de espécies apresentou um rápido aumento conforme o esforço amostral. A curva tendeu a uma assíntota, mas provavelmente mais espécies devem ser encontradas com maiores esforços na área, uma vez que a assíntota plena não foi atingida. Com base no estimador Bootstrap, a riqueza estimada para a área de estudo é de aproximadamente 11 espécies (Figura 4).

\section{Discussão}

Os resultados encontrados no presente estudo corroboram outros estudos realizados focados na distribuição local de lagartos em áreas de Cerrado (Colli et al. 2002, Nogueira et al. 2005, 2009), onde as Matas de galeria abrigaram menor número de espécies quando comparada com áreas abertas. De acordo com Colli et al. (2002) e Rodrigues (2005b), as Matas de galeria não apresentam fauna própria específica e muitas espécies encontradas nos Cerrados abertos também utilizam essas áreas.

Entre as cinco espécies registradas nas Matas de galeria, somente a espécie $H$. imbricatus não foi registrada na área de Cerrado, sendo aparentemente dependente de habitats florestais (Figura 3, Tabela 1). Essa espécie está restrita a áreas de clima mais frio associadas com altas elevações e áreas montanhosas do leste do Brasil (Rodrigues et al. 2009) e geralmente é encontrada na serrapilheira (Dixo \& Verdade 2006). Inicialmente, esta espécie estava restrita a áreas de Mata Atlântica e posteriormente Novelli et al. (2010) registraram a sua ocorrência no domínio do bioma Cerrado.

A espécie $U$. vautieri foi registrada pela primeira vez em uma área de mata na região do Cerrado na RBUB (Santos et al. 2009), ampliando sua distribuição geográfica para este domínio. Embora esta espécie fosse conhecida somente para a Mata Atlântica (Etheridge \& Williams 1991, Pellegrino et al. 1999), no presente trabalho foram registrados indivíduos na área de Cerrado sensu stricto. Sua associação com o Cerrado pode estar relacionada ao fato de várias áreas naturais desse bioma no sul de Minas Gerais se encontrarem em áreas transicionais com áreas isoladas de Mata Atlântica no Estado.

No Cerrado, $M$. dorsivittata, é mais abundante em ambientes de campo limpo, campo sujo e bordas de campo úmido, geralmente com densa cobertura de gramíneas (Nogueira et al. 2005). É uma espécie típica de formações abertas, habitando preferencialmente fisionomias campestres (Valdujo 2003, Nogueira et al. 2005). Sua associação com áreas de Cerrado nesse estudo esteve mais relacionada com áreas abertas, assim como relatado também por Nogueira et al. (2005) e Sousa et al. (2010), podendo mais raramente ocorrer também em hábitats florestados, como matas de galeria (Colli et al. 2002). Mabuya frenata foi a única espécie encontrada em todas as fitofisionomias. Esta espécie ocorre em áreas abertas na América do Sul, nos biomas Cerrado e Chaco (Vanzolini 1988) e no Brasil, nas regiões Centro-Oeste e Sudeste (Peters \& Donoso-Barros 1986). Apenas um espécime foi coletado no campo rupestre sobre uma rocha (talvez devido à dificuldade de uma maior amostragem nesta fitofisionomia), mas segundo Vrcibradic \& Rocha (1998) esses ambientes são altamente utilizados por esta espécie. Esta espécie também está associada a áreas de Mata Atlântica no Rio de Janeiro (Vrcibradic et al. 2006) e em Minas Gerais (Costa et al. 2008).

A maioria das espécies distribuídas dentro da região do Cerrado ocorre principalmente em áreas com vegetação aberta, como no caso das espécies $A$. ameiva e $P$. acutirostris, que são espécies de ampla distribuição geográfica. Polychrus acutirostris no Brasil pode ser encontrado nas regiões Nordeste, Sul e Centro-Oeste e também nos Estados de São Paulo, Minas Gerais e no sul do Pará. (Vitt \& Lacher 1981; Vanzolini 1983; Nogueira et al. 2005; Recorder \& Nogueira 2007). A maioria dos espécimes de P. acutirostris capturados neste estudo foi coletada nas armadilhas de interceptação e queda, constatando que a espécie pode ser encontrada no chão, como também observado por Vitt \& Lacher (1981). Enyalius bilineatus é encontrada no sudeste e leste de Minas Gerais (Jackson 1978, Sousa et al. 2010) e é a única espécie do gênero Enyalius associada ao Cerrado, em áreas florestadas, principalmente em matas de galeria (Bertolotto et al. 2002, Colli et al. 2002, Bertoluci et al. 2009, Sousa et al. 2010. Contudo, neste trabalho apenas um indivíduo de E. bilineatus foi registrado em matas de galeria, sendo em sua maioria registrados no Cerrado sensu stricto. O tipo de habitat fossorial encontrado em algumas espécies dificulta na amostragem destas, como observado para a espécie Ophiodes striatus em área de Cerrado. Colli et al. (2002) também constataram a presença dessa espécie em áreas de Cerrado, porém sempre associada a áreas florestadas e, na região core

Tabela 1. Composição da fauna de lagartos da Reserva Biológica UnilavrasBoqueirão. Fitofisionomias: $\mathrm{CE}=$ Cerrado sensu stricto; $\mathrm{MA}=$ Mata de Galeria e CR = Campo Rupestre.

Table 1. Composition of lizard fauna from Reserva Biológica UnilavrasBoqueirão. Physionomies: $\mathrm{CE}=$ Cerrado sensu stricto; $\mathrm{MA}=$ Gallery Forest and $\mathrm{CR}=$ Campo Rupestre

\begin{tabular}{|c|c|c|c|c|}
\hline \multirow{2}{*}{ Táxon } & \multirow{2}{*}{$\mathbf{N}$} & \multicolumn{3}{|c|}{ Área } \\
\hline & & $\mathbf{C E}$ & MA & $\mathbf{C R}$ \\
\hline \multicolumn{5}{|l|}{ SQUAMATA - SAURIA } \\
\hline \multicolumn{5}{|l|}{ ANGUIDAE } \\
\hline Ophiodes striatus (Spix, 1824) & 1 & 1 & & \\
\hline \multicolumn{5}{|l|}{ GYMNOPHTHALMIDAE } \\
\hline Heterodactylus imbricatus Spix, 1825 & 2 & & 2 & \\
\hline Cercosaura ocellata Wagler, 1830 & 2 & 1 & & 1 \\
\hline \multicolumn{5}{|l|}{ LEIOSAURIDAE } \\
\hline Enyalius bilineatus Duméril \& Bibron, 1837 & 24 & 23 & 1 & \\
\hline Urostrophus vautieri Duméril \& Bibron, 1837 & 8 & 3 & 5 & \\
\hline \multicolumn{5}{|l|}{ POLYCHROTIDAE } \\
\hline Polychrus acutirostris Spix, 1825 & 15 & 15 & & \\
\hline \multicolumn{5}{|l|}{ SCINCIDAE } \\
\hline Mabuya frenata (Cope, 1862) & 9 & 6 & 2 & 1 \\
\hline Mabuya dorsivittata Cope, 1862 & 6 & 5 & 1 & \\
\hline \multicolumn{5}{|l|}{ TEIIDAE } \\
\hline Ameiva ameiva (Linnaeus, 1758) & 7 & 7 & & \\
\hline \multicolumn{5}{|l|}{ TROPIDURIDAE } \\
\hline Tropidurus itambere Rodrigues, 1987 & 11 & 1 & & 10 \\
\hline Número de indivíduos & 85 & 62 & 11 & 12 \\
\hline
\end{tabular}


do Cerrado, é principalmente encontrada em formações de campos (Nogueira et al. 2009).

Apesar do esforço dedicado na verificação da composição e riqueza de lagartos do Cerrado, os estudos para o estado de Minas Gerais são ainda incipientes quando comparados aos realizados em outras áreas de Cerrado como no Centro-oeste do Brasil. Dentre as espécies registradas, $T$. itambere estava frequentemente associado a lugares com afloramentos rochosos, confirmando a observação de Rodrigues (1987) e de Colli et al. (2002), de que esta espécie é típica de áreas abertas de Cerrado. O mesmo padrão também foi observado por Ávila Pires (1995) e Sousa et al. (2010) para C. ocellata, que pode habitar formações savânicas e áreas marginais nas formações
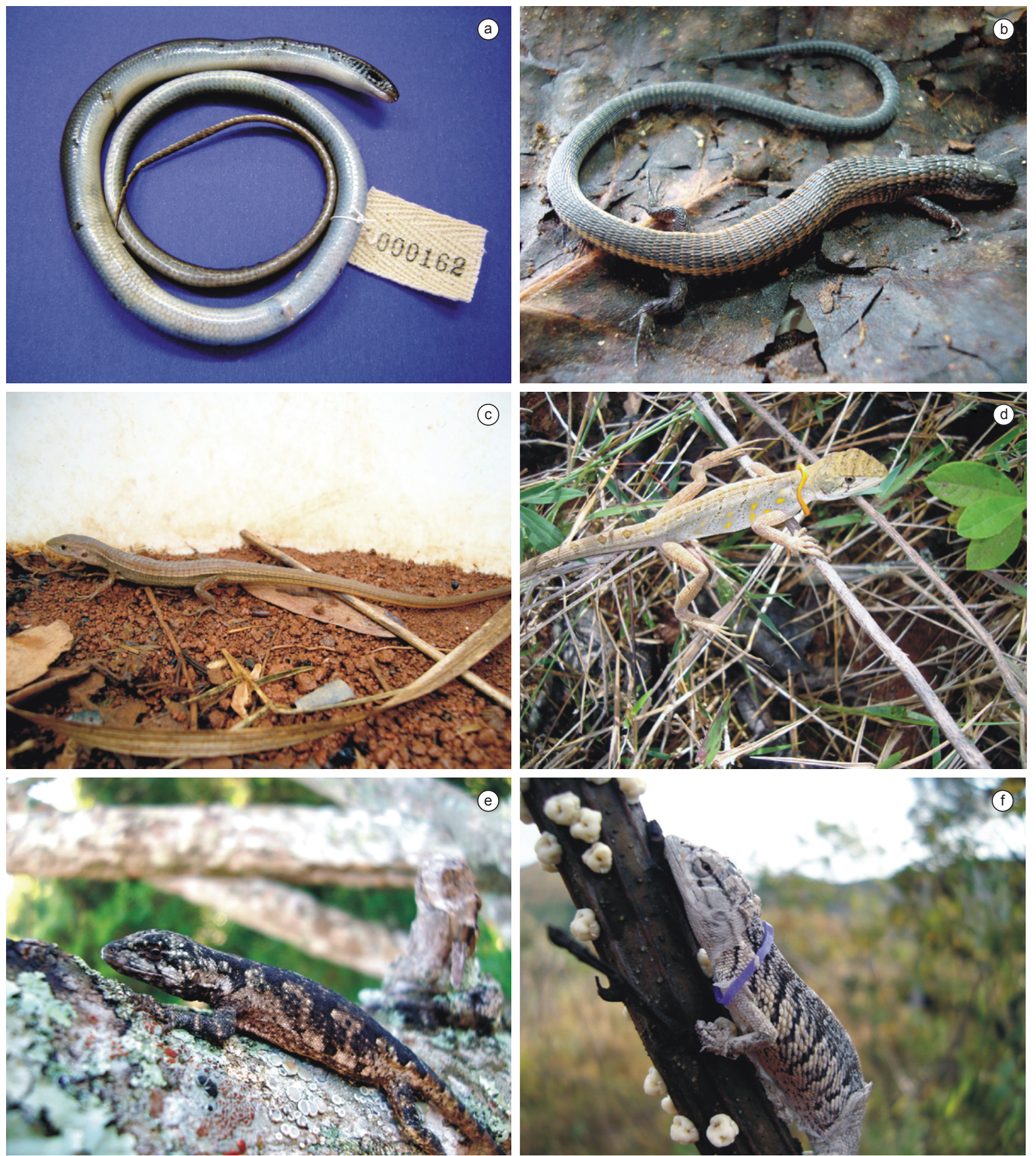

Figura 1. Espécies de lagartos registradas na Reserva Biológica Unilavras - Boqueirão, no município de Ingaí, Estado de Minas Gerais, Brasil. a) Ophiodes striatus; b) Heterodactylus imbricatus; c) Cercosaura ocellata; d) Enyalius bilineatus; e) Urostrophus vautieri; f) Polychrus acutirostris.

Figure 1. Lizard species recorded in Reserva Biológica Unilavras - Boqueirão, municipality of Ingaí, Minas Gerais State, Brazil. a) Ophiodes striatus; b) Heterodactylus imbricatus; c) Cercosaura ocellata; d) Enyalius bilineatus; e) Urostrophus vautieri; f) Polychrus acutirostris. 

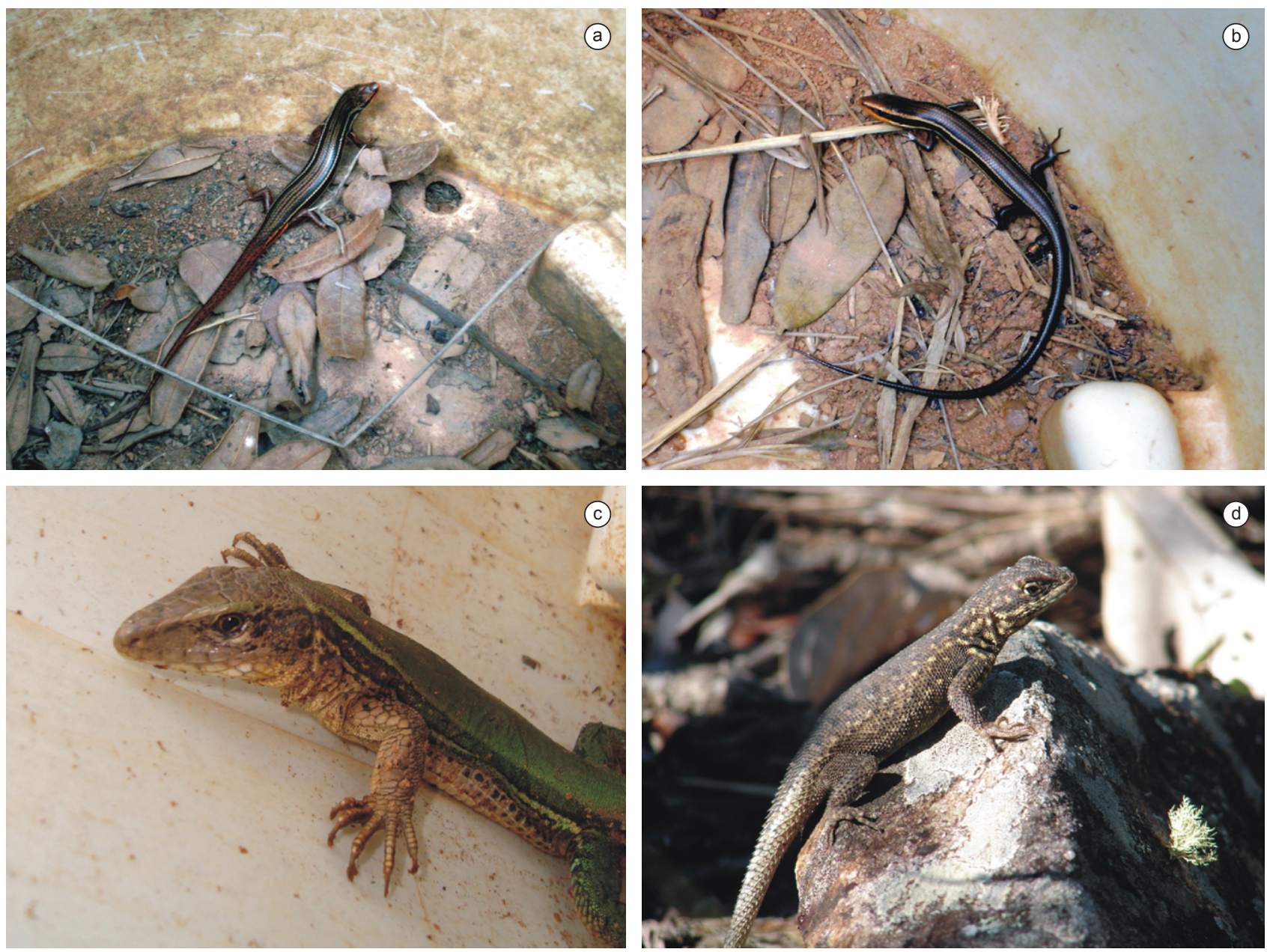

Figura 2. Espécies de lagartos registradas na Reserva Biológica Unilavras - Boqueirão, no município de Ingaí, Estado de Minas Gerais, Brasil. a) Mabuya dorsivittata; b) Mabuya dorsivittata; c) Ameiva ameiva; d) Tropidurus itambere.

Figure 2. Lizard species recorded in Reserva Biológica Unilavras - Boqueirão, municipality of Ingaí, Minas Gerais State, Brazil. a) Mabuya frenata; b) Mabuya dorsivittata; c) Ameiva ameiva; d) Tropidurus itambere.

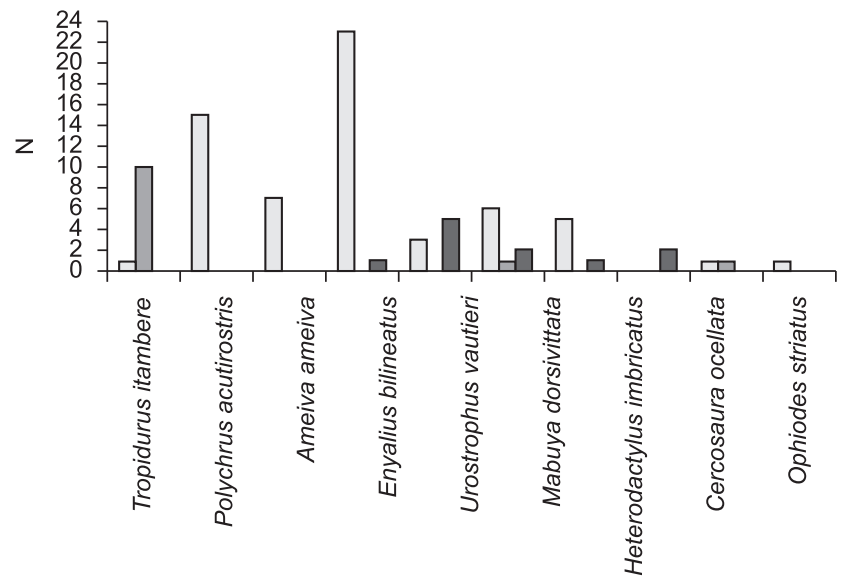

$\square$ Cerrado sensu stricto $\square$ Campo rupestre $\square$ Mata de galeria

Figura 3. Número de espécimes $(\mathrm{N})$ de lagartos coletadas nas três áreas na Reserva Biológica Unilavras-Boqueirão: Cerrado sensu stricto, Campo Rupestre e Mata de Galeria.

Figure 3. Number of lizard specimens $(\mathrm{N})$ collected in three areas in the Reserva Biológica Unilavras-Boqueirão: Cerrado sensu stricto, rocky outcrops and gallery forests. florestais. Neste estudo um espécime de C. ocellata foi encontrado em vegetação associada a rochas nos campos rupestres.

As espécies de lagartos registradas na Reserva Biológica Unilavras-Boqueirão correspondem a 17,5\% das 57 espécies conhecidas para o Cerrado de Minas Gerais, segundo Bérnils et al. (2009). Com base na curva de rarefação de espécies, pode-se afirmar que os dados obtidos da fauna de lagartos da RBUB são representativos localmente, considerando que a curva de rarefação estimada indica uma riqueza potencial de 11 espécies. Em Minas Gerais, Recorder \& Nogueira (2007) registraram 25 espécies de lagartos em uma área de Cerrado do Parque Nacional Grande Sertão Veredas, na divisa dos Estados de Minas Gerais e Bahia e Sousa et al. (2010), em uma amostragem mais recente em fragmentos de Cerrado e Mata Atlântica no Campo das Vertentes, no município de Ritápolis, Minas Gerais, registraram a ocorrência de nove espécies de lagartos. Essas desigualdades podem estar relacionadas com os diferentes métodos de amostragem, tempo e diferentes esforços amostrais empregados na captura de lagartos. Com base nos números, sabe-se que o Cerrado abriga maior diversidade da fauna de lagartos quando comparada com a Mata Atlântica. Sabe-se também que prováveis diferenças na riqueza da fauna de lagartos no Cerrado podem estar mais associadas com os diferentes processos regionais, do que 


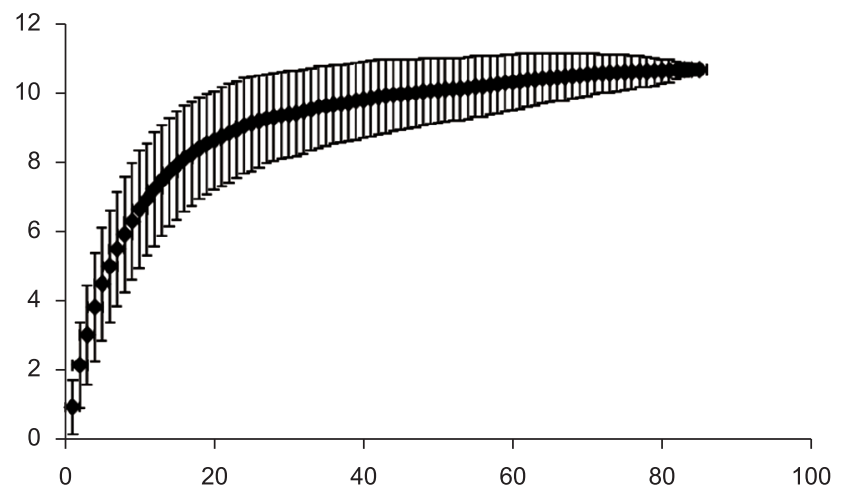

Figura 4. Curva de rarefação das espécies de lagartos amostradas na Reserva Biológica do Unilavras - Boqueirão, Ingaí, Minas Gerais.

Figure 4. Rarefaction curve of lizard species sampled in Reserva Biológica do Unilavras - Boqueirão, Ingaí, Minas Gerais.

propriamente dentro das fitofisionomias que caracterizam uma única localidade (Nogueira et al. 2009).

A escassez de estudos que caracterizam a composição da fauna de lagartos do estado de Minas Gerais pode ser apontada como uma das causas da presença de um grande número de espécies de répteis na categoria "Dados Deficientes" na Lista de Espécies Ameaçadas de Extinção da Fauna do estado (Conselho... 2008). Os registros provenientes da RBUB indicam a necessidade de ampliar os estudos para demais áreas do estado de Minas Gerais, avaliando o potencial para a ocorrência de espécies ou a ampliação da ocorrência de espécies de lagartos então desconhecidas no Cerrado, servindo como ferramentas que irão ajudar na escolha de áreas prioritárias e a criação de Unidades de Conservação neste hotspot de biodiversidade.

\section{Agradecimentos}

Ao Instituto Brasileiro do Meio Ambiente e dos Recursos Naturais Renováveis (IBAMA/SISBIO) pela permissão de coleta e transporte dos espécimes (Registros 1971350 e 1971350). À Fundação de Amparo à Pesquisa do Estado de Minas Gerais (PROBIC/ FAPEMIG) pelo financiamento do projeto do qual esse estudo faz parte. Ao Dr. Fernando Antônio Frieiro-Costa pela ajuda na escolha e estabelecimento das áreas de coleta. Ao Sr. Fabiano B. Silva pela ajuda nos trabalhos de campo.

\section{Referências Bibliográficas}

ASSIS, V.B. 1999. Introdução às serpentes da Serra do Cipó (municípios de Santana do Riacho e Jaboticatubas). Bios 7(7):69-71.

ÁVILA PIRES, T.C.S. 1995. Lizards of Brazilian Amazonia (Reptilia: Squamata). Zoologische Verhandelingen, Leiden.

BARROS FILHO, J.D., SOUSA, B.M. \& CARVALHO, R.M.H. 2005. Amphisbaena dubia (NCN). Herpetol. Rev. 36(3):335-335.

BAUER, A.M. \& SADLIER. R.A. 1992. The use of mouse glue traps to capture lizards. Herpetol. Rev. 23(4):112-113.

BERNARDE, P.S. 2008. Ecologia e métodos de amostragem de Répteis Squamata. In VIII Congresso Internacional Sobre Manejo de Fauna Silvestre na Amazônia e América Latina (F.P.C. Silva, D.A.P. Gomes-Silva, J.S. Melo, V.M.L. Nascimento, coords.). Coletânea de textos - Manejo e Monitoramento de Fauna Silvestre em Florestas Tropicais, Rio Branco, p.189-201.

BÉRNILS, R.S., NOGUEIRA, C.C. \& XAVIER-DA-SILVA, V. 2009. Répteis. In Biota Minas: diagnóstico do conhecimento sobre a biodiversidade no Estado de Minas Gerais (G.M. Drummond, C.S. Martins, M.B. Greco \& F. Vieira, eds.). Fundação Biodiversitas, Belo Horizonte, p.251-278.
BÉRNILS, R.S. 2010. Brazilian Reptiles - List of Species. Sociedade Brasileira de Herpetologia. http://www.sbherpetologia.org.br/checklist/ repteis.htm (último acesso em 16/11/2010).

BERTOLOTTO, C.E. PELLEGRINO, V.K.C., RODRIGUES, M.T. \& YONENAGA-YASSUDA, Y. 2002. Comparative cytogenetics and supernumerary chromosomes in the Brazilian lizards genus Enyalius (Squamata, Polichrotidae). Hereditas 136:51-57. PMid:12184489.

BERTOLUCI, J., CANELAS, M.A.S., EISEMBERG, C.C., PALMUTI, C.F.S. \& MONTINGELLI, G.G. 2009. Herpetofauna of Estação Ambiental de Peti, an Atlantic Rainforest fragment of Minas Gerais State, southeastern Brazil. Biota Neotrop. 9(1): http://www.biotaneotropica.org.br/v9n1/en/a bstract?inventory+BN01409012009 (último acesso em 22/07/2010).

BRITES, V.L.C. \& BAUAB, F.A. 1988. Fauna ofidiana do município de Uberlândia, Minas Gerais - Brasil. I. Ocorrência na área urbana. Rev. Cent. Cienc. Biomed. Univ. Fed. Uberlandia 4(1):3-8.

CECHIN, S.Z. \& MARTINS, M. 2000. Eficiência de armadilhas de queda (pitfall traps) em amostragens de anfíbios e répteis no Brasil. Rev. Bras. Zool. 17(3):729-740.

COLLI, G.R., BASTOS, R.P. \& ARAÚJO, A.F.B. 2002. The character and dynamics of the Cerrado herpetofauna. In The Cerrados of Brazil: ecology and natural history of a Neotropical Savanna (P.S. Oliveira \& R.J. Marquis, eds.). Columbia University Press, New York, p.223-241.

COLWELL, R.K. 2006. EstimateS: Statistical estimation of species richness and shared species from samples. version 8. User's Guide and application. http:// purl.oclc.org/estimates.

CONSELHO DE POLÍTICA AMBIENTAL - COPAM, 2008. Deliberação $\mathrm{COPAM} \mathrm{n}^{\circ} 366$, de 15 de dezembro de 2008. Lista de Espécies Ameaçadas de Extinção da Fauna do Estado de Minas. http://www.ief.mg.gov.br/ images/stories/biodiversidade/faunaameacada_dn366_2008 (último acesso em 01/072010).

COSTA, H.C., FERNANDES, V.D., VRCIBRADIC, D. \& FEIO, R.N. 2008. Notes on Geographic Distribution: Reptilia, Scincidae, Mabuya frenata: Distribution extension. Check List 4(1):86-88.

DIXO, M. \& VERDADE, V.K. 2006. Herpetofauna de serrapilheira da Reserva Florestal de Morro Grande, Cotia (SP). Biota Neotrop. 6(2): http://www. biotaneotropica.org.br/v6n2/pt/abstract?article+bn00806022006 (último acesso em 30/06/2010).

DRUMMOND, G.M., MARTINS, C.S., MACHADO, A.B.M., SEBAIO, F.A. \& ANTONINI, Y. 2005. Biodiversidade em Minas Gerais: um Atlas para sua conservação. 2. ed. Fundação Biodiversitas, Belo Horizonte.

DRUMMOND, G.M., MARTINS, C.S., GRECO, M.B., VIEIRA, F. 2009. Biota Minas: diagnóstico do conhecimento sobre a biodiversidade no Estado de Minas Gerais subsídio ao Programa Biota Minas. Fundação Biodiversitas, Belo Horizonte.

ENGE, K.M. 2001.The Pitfalls of Pitfall Traps. J. Herpetol. 35(3):467-478.

ETHERIDGE, R. \& WILliAMS, E.E. 1991. A review of the South American lizard genera Urostrophus and Anisolepis (Squamata: Iguania: Polychridae). Bull. Mus. Comp. Zool. 152(5):317-361.

HEYER, W.R., DONNELY, R.W., McDIARMIND, R.W., HAYEK, L.C. \& FOSTER, M.S. 1994. Measuring and monitoring biological diversity: standard methods for amphibians. Smithsonian Institution Press, Washington.

JACKSON, J.F. 1978. Differentiation in the genera Enyalius and Strobilurus (Iguanidae): implications for pleistocene climatic changes in eastern Brazil. Arq. Zool. 30(1):1-79.

MESQUITA, D.O., COLLI, G.R. \& VITT, L.J. 2007. Ecological release in lizard assemblages of neotropical savanna. Oecologia 153(1):185-195.

MITTERMEIER, R.A., GIL, P.R., HOFMANN, M., PILGRIM, J., BROOKS, T., MITTERMEIER, C.G., LAMOREAUX, J. \& FONSECA, G.A.B. 2004. Hotspots revisited: Earth's biologically richest and most endangered terrestrial ecoregions. Cemex, Washington.

NOGUEIRA, C., COLLI, G.R. \& MARTINS, M. 2009. Local richness and distribution of the lizard fauna in natural habitat mosaics of the Brazilian Cerrado. Aust. Ecol. 34:83-96. 
NOGUEIRA, C., RiBeiRO, S., COSTA, G.C. \& COLLI, G.R. 2011 Vicariance and endemism in a Neotropical savanna hotspot: distribution patterns of Cerrado squamate reptiles. J. Biogeogr. 38:1907-1922. http:// dx.doi.org/10.1111/j.1365-2699.2011.02538.x

NOGUEIRA, C., VALDUJO, H. \& FRANÇA, F.G.R. 2005. Habitat variation and lizard diversity in a Cerrado área of Central Brazil. Stud. Neot. Faun. Envir. 40(2):105-112.

NOVELLI, I.A., LUCAS, P.S., SANTOS, R.C. 2010. Reptilia, Squamata, Gymnophthalmidae, Heterodactylus imbricatus: Distribution extension. CheckList. 7(1):30-31.

PELLEGRINO, K.C., BERTOLOTTO, C.E.V., RODRIGUES, M.T. \& YONENAGA-YASSUDA, Y. 1999. Banding patterns, heteromorphic sex chromosomes and Ag-stained NORs after pachytene stages in the meiosis of the Brazilian lizard Urostrophus vautieri (Squamata, Polychrotidae). Caryologia 52(1-2): 21-26.

PEREIRA, G.J.M. \& VOLPATO, M.M.L. 2005. Levantamento das características bióticas e abióticas da área do Boqueirão. Pro Homine 4:27-34.

PETERS, A. J., DONOSO-BARROS, R. \& OREJAS-MIRANDA, B. 1986. Catalogue of neotropical squamata Part I: Snakes - Part II: Lizards and amphisbaenians. with new material by P. E. Vanzolini. Smithsonian Instituion Press, Washington and London.

RECORDER, R. \& NOGUEIRA, C. 2007. Composição e diversidade de répteis na região sul do Parque Nacional Grande Sertão Veredas, Brasil Central. Biota Neotrop. 7(3): http://www.biotaneotropica.org.br/v7n3/ pt/abstract?inventory+bn01107032007 (último acesso em 19/10/2010).

RIBEIRO, L.B. \& SOUSA, B.M. 2006. Elastic Hair Bands: An Effective Marking Technique for Lizards in Mark-Recapture Studies. Herpetol. Rev. 37 (4):434-435.

RODRIGUES, M.T. 1987. Sistemática, ecologia e zoogeografia dos Tropidurus do grupo torquatus ao Sul do Rio Amazonas (Sauria, Iguanidae). Arq. Zool. 31(3):105-230.

RODRIGUES, M.T. 2005a. Conservação dos répteis brasileiros: os desafios para um país megadiverso. Megadiversidade 1(1):87-94.

RODRIGUES, M.T. 2005b. A biodiversidade dos Cerrados: conhecimento atual e perspectivas, com uma hipótese sobre o papel das matas de galerias na troca faunística durante ciclos climáticos. In Cerrado: Ecologia, Biodiversidade e Conservação (A. Scariot, J.C. Santos-Silva \& J.M. Felfili, eds.). Ministério do Meio Ambiente, Brasília, p.236-246.
RODRIGUES, M.T., FREITAS, M.A. \& SILVA, T.F.S. 2009. New Species of Earless Lizard Genus Heterodactylus (Squamata: Gymnophthalmidae) from the Highlands of Chapada Diamantina, State of Bahia, Brazil. J. Herpetol. 43(4):605-611. http://dx.doi.org/10.1670/08-133.1

SANTOS, R.C., LUCAS, P.S., SOUSA, B.M. \& NOVELLI, I.A. 2009 Reptilia, Squamata, Leiosauridae, Urostrophus vautieri: Distribution extension and geographic distribution map. CheckList 5(3):533-536.

SÃO PEDRO, V.A. \& PIRES, M.R. 2009. As Serpentes da Região de Ouro Branco, extremo sul da Cadeia do Espinhaço, Minas Gerais. Rev. Ceres 56(2):166-171.

SOUSA, B.M. \& NOVELLI, I.A. 2009. Reptilia, Testudines, Chelidae: Hydromedusa tectifera: Distribution extension in Brazil. CheckList 5(3):396-398.

SOUSA, B.M., NASCIMENTO, A.E.R., GOMIDES, S.C., VARELA RIOS, C.H., HUDSON, A.H. \& NOVELLI, I.A. 2010. Reptiles in fragments of Cerrado and Atlantic Forest at the Campo das Vertentes, Minas Gerais State, Southeastern Brazil. Biota Neotrop. 10(2):http://www. biotaneotropica.org.br/v10n2/pt/abstract?article+bn03510022010 (ultimo acesso em 02/11/2010).

UETZ, P. \& HALLERMANN, J. 2010. How Many Species? Base de dados eletrônica acessível em: http://www.reptiledatabase. org/db-info/ SpeciesStat.html (último acesso em 27/05/2010).

VALDUJO, P.H. 2003. Distribuição da comunidade de lagartos no mosaico fisionômico do Cerrado e suas implicações para conservação. Dissertação de Mestrado, Universidade de Brasília, Distrito Federal.

VANZOLINI. P.E. 1988. Distributional patterns of South American lizards. In: Proceedings of a workshop on Neotropical distributional patterns (P.E. Vanzolini \& W.R. Heyer, eds.). Academia Brasileira de Ciências, Rio de Janeiro, p.317-342.

VANZOLINI, P. E. 1983. Guiano - Brasilian Polychrus: distribuition and apeciation (Sauria, Iguanidae). Adv. Herpet. Evol. Biol. 2(1):118-131.

VITT, L.J. \& LACHER, T.E. 1981. Behaviour, habitat, diet and reproduction of the iguanid lizard Polychrus acutirostris in the Caatinga of northeastern Brazil. Herpetologica 37(1):53-63.

VRCIBRADIC, D. \& ROCHA, C.F.D. 1998. The ecology of skink Mabuya frenata in an area of rock outcrops in Southeastern Brazil. J. Herpetol. 32(2):229-237.

VRCIBRADIC, D., ALMEIDA GOMES, M., BORGES JUNIOR, V.N., KIEFER, M.C., VAN SLUYS, M. \& ROCHA, C.F.D. 2006. Reptilia, Scincidae, Mabuya frenata: Distribution extension. Check List 2(2):57-58.

ZANI, P.A. \& VITT, L.J. 1995. Techniques for capturing arboreal lizards. Herpetol. Rev. 26:136-137 